Stud. Univ. Babeş-Bolyai Math. 62(2017), No. 2, 217-232

DOI: 10.24193/subbmath.2017.2.08

\title{
Mellin transform in bicomplex space and its application
}

\author{
Ritu Agarwal, Mahesh Puri Goswami and Ravi P. Agarwal
}

\begin{abstract}
Motivated by the recent applications of bicomplex theory to the study of functions of large class, in this paper, we define bicomplex Mellin transform of bicomplex-valued functions. Also, we derive some of it's basic properties and inversion theorem in bicomplex space. Application of bicomplex Mellin transform in networks with time-varying parameters problem has been illustrated.
\end{abstract}

Mathematics Subject Classification (2010): 30G35, 42B10.

Keywords: Bicomplex functions, bicomplex numbers, bicomplex Laplace transform, Mellin transform.

\section{Introduction}

In this paper, we extend the Mellin transform of complex-valued function in complex variable to Mellin transform of bicomplex-valued function in bicomplex variable. In 1892, Segre Corrado [18] defined bicomplex numbers as

$$
C_{2}=\left\{\xi: \xi=x_{0}+i_{1} x_{1}+i_{2} x_{2}+j x_{3} \mid x_{0}, x_{1}, x_{2}, x_{3} \in C_{0}\right\},
$$

or

$$
C_{2}=\left\{\xi: \xi=z_{1}+i_{2} z_{2} \mid z_{1}, z_{2} \in C_{1}\right\} .
$$

where $i_{1}$ and $i_{2}$ are imaginary units such that $i_{1}^{2}=i_{2}^{2}=-1, i_{1} i_{2}=i_{2} i_{1}=j, j^{2}=1$ and $C_{0}, C_{1}$ and $C_{2}$ are sets of real numbers, complex numbers and bicomplex numbers, respectively. The set of bicomplex numbers is a commutative ring with unit and zero divisors. Hence, contrary to quaternions, bicomplex numbers are commutative with some non-invertible elements situated on the null cone.

In 1928 and 1932, Futagawa Michiji originated the concept of holomorphic functions of a bicomplex variable in a series of papers [10], [11]. In 1934, Dragoni [8] gave some basic results in the theory of bicomplex holomorphic functions while Price G.B. [16] and Rönn S. [17] have developed the bicomplex algebra and function theory. 
In recent developments, authors have done efforts to extend Polygamma function [13], inverse Laplace transform, it's convolution theorem [2], Stieltjes transform [1], Tauberian Theorem of Laplace-Stieltjes transform [3] and Bochner Theorem of Fourier-Stieltjes transform [4] in the bicomplex variable from their complex counterpart. In their procedure, the idempotent representation of bicomplex numbers plays a vital role.

Hjalmar Mellin (1854-1933, see, e.g. [15]) gave his name to the Mellin transform that associates to a complex-valued function $f(t)$ defined over the interval $(0, \infty)$, the function of complex variable $s$, as

$$
\bar{f}(s)=\int_{0}^{\infty} t^{s-1} f(t) d t .
$$

The change of variables $t=e^{-x}$ shows that the Mellin transform is closely related to the Laplace transform. General properties of the Mellin transform are usually treated in detail in books on integral transforms, like those of Poularikas A.D. [15] and Davies B. [6]. In 1959, Francis R.G. [12] discussed the application of complex Mellin transform to networks with time-varying parameters. In 1995, Flajolet P. et al. [9] used Mellin transform for the asymptotic analysis of harmonic sums.

For solving the large class of bicomplex partial differential equations, we need integral transforms defined for large class. In this process we derive bicomplex Mellin transform with convergence conditions that can be capable of transferring the signals from real-valued $t$ domain to bicomplexified frequency $\xi$ domain.

Idempotent Representation: Every bicomplex number can be uniquely expressed as a complex combination of $e_{1}$ and $e_{2}$, viz.

$$
\xi=\left(z_{1}+i_{2} z_{2}\right)=\left(z_{1}-i_{1} z_{2}\right) e_{1}+\left(z_{1}+i_{1} z_{2}\right) e_{2},
$$

(where $e_{1}=\frac{1+j}{2}, e_{2}=\frac{1-j}{2} ; e_{1}+e_{2}=1$ and $e_{1} e_{2}=e_{2} e_{1}=0$ ).

This representation of a bicomplex number is known as Idempotent Representation of $\xi$. The coefficients $\left(z_{1}-i_{1} z_{2}\right)$ and $\left(z_{1}+i_{1} z_{2}\right)$ are called the Idempotent Components of the bicomplex number $\xi=z_{1}+i_{2} z_{2}$ and $\left\{e_{1}, e_{2}\right\}$ is called Idempotent Basis.

Cartesian Set: The Auxiliary complex spaces $A_{1}$ and $A_{2}$ are defined as follows:

$$
A_{1}=\left\{w_{1}=z_{1}-i_{1} z_{2}, \forall z_{1}, z_{2} \in C_{1}\right\}, A_{2}=\left\{w_{2}=z_{1}+i_{1} z_{2}, \forall z_{1}, z_{2} \in C_{1}\right\} .
$$

A cartesian set $X_{1} \times_{e} X_{2}$ determined by $X_{1} \subseteq A_{1}$ and $X_{2} \subseteq A_{2}$ and is defined as:

$$
X_{1} \times_{e} X_{2}=\left\{z_{1}+i_{2} z_{2} \in C_{2}: z_{1}+i_{2} z_{2}=w_{1} e_{1}+w_{2} e_{2}, w_{1} \in X_{1}, w_{2} \in X_{2}\right\} .
$$

With the help of idempotent representation, we define projection mappings $P_{1}: C_{2} \rightarrow$ $A_{1} \subseteq C_{1}, P_{2}: C_{2} \rightarrow A_{2} \subseteq C_{1}$ as follows:

$P_{1}\left(z_{1}+i_{2} z_{2}\right)=P_{1}\left[\left(z_{1}-i_{1} z_{2}\right) e_{1}+\left(z_{1}+i_{1} z_{2}\right) e_{2}\right]=\left(z_{1}-i_{1} z_{2}\right) \in A_{1}, \forall z_{1}+i_{2} z_{2} \in C_{2}$, $P_{2}\left(z_{1}+i_{2} z_{2}\right)=P_{2}\left[\left(z_{1}-i_{1} z_{2}\right) e_{1}+\left(z_{1}+i_{1} z_{2}\right) e_{2}\right]=\left(z_{1}+i_{1} z_{2}\right) \in A_{2}, \forall z_{1}+i_{2} z_{2} \in C_{2}$.

In the following theorem, Price G.B. discussed the convergence of bicomplex function with respect to it's idempotent complex component functions. This theorem is useful in proving our results. 
Theorem 1.1. (Price G.B. [16]). $F(\xi)=F_{e_{1}}\left(\xi_{1}\right) e_{1}+F_{e_{2}}\left(\xi_{2}\right) e_{2}$ is convergent in domain $D \subseteq C_{2}$ iff $F_{e_{1}}\left(\xi_{1}\right)$ and $F_{e_{2}}\left(\xi_{2}\right)$ under functions $P_{1}: D \rightarrow D_{1} \subseteq C_{1}$ and $P_{2}: D \rightarrow$ $D_{2} \subseteq C_{1}$ are convergent in domains $D_{1}$ and $D_{2}$, respectively.

The organization of this paper is as follows:

In Section 2, we establish bicomplex Mellin transform with convergence conditions. In Section 3, we present some useful properties of bicomlex Mellin transform. In Section 4, we establish the inversion theorem for bicomplex Mellin transform. In section 5, we discuss application of bicomplex Mellin transform in finding the solution of bicomplex partial differential equation generated by network model and last Section 6 contains the conclusion.

\section{Bicomplex Mellin transform}

Let $f_{1}(t)$ be a complex-valued continuous function on the interval $(0, \infty)$ with $f_{1}(t)=O\left(t^{-\alpha_{1}}\right)$ as $t \rightarrow 0^{+}$and $f_{1}(t)=O\left(t^{-\beta_{1}}\right)$ as $t \rightarrow \infty$, where $\alpha_{1}<\beta_{1}$. Then Mellin transform of $f_{1}(t)$ is

$$
\mathfrak{M}\left[f_{1}(t) ; s_{1}\right]=\int_{0}^{\infty} t^{s_{1}-1} f_{1}(t) d t=\bar{f}_{1}\left(s_{1}\right), \quad s_{1} \in C_{1}
$$

where $\bar{f}_{1}\left(s_{1}\right)$ is analytic and convergent in the vertical strip

$$
\Omega_{1}=\left\{s_{1} \in C_{1}: \alpha_{1}<\operatorname{Re}\left(s_{1}\right)<\beta_{1}\right\} .
$$

Similarly, $f_{2}(t)$ be a complex-valued continuous function on the interval $(0, \infty)$ with $f_{2}(t)=O\left(t^{-\alpha_{2}}\right)$ as $t \rightarrow 0^{+}$and $f_{2}(t)=O\left(t^{-\beta_{2}}\right)$ as $t \rightarrow \infty$, where $\alpha_{2}<\beta_{2}$. Then Mellin transform of $f_{2}(t)$ is

$$
\mathfrak{M}\left[f_{2}(t) ; s_{2}\right]=\int_{0}^{\infty} t^{s_{2}-1} f_{2}(t) d t=\bar{f}_{2}\left(s_{2}\right), \quad s_{2} \in C_{1}
$$

where $\bar{f}_{2}\left(s_{2}\right)$ is analytic and convergent in the vertical strip

$$
\Omega_{2}=\left\{s_{2} \in C_{1}: \alpha_{2}<\operatorname{Re}\left(s_{2}\right)<\beta_{2}\right\} .
$$

Since $\bar{f}_{1}\left(s_{1}\right)$ and $\bar{f}_{2}\left(s_{2}\right)$ are complex functions which are analytic and convergent in the strips $\Omega_{1}$ and $\Omega_{2}$ respectively. Now, we have linear combination of $\bar{f}_{1}\left(s_{1}\right)$ and $\bar{f}_{2}\left(s_{2}\right)$ w.r.t. $e_{1}$ and $e_{2}$ respectively

$$
\begin{aligned}
\bar{f}_{1}\left(s_{1}\right) e_{1}+\bar{f}_{2}\left(s_{2}\right) e_{2} & =\left(\int_{0}^{\infty} t^{s_{1}-1} f_{1}(t) d t\right) e_{1}+\left(\int_{0}^{\infty} t^{s_{2}-1} f_{2}(t) d t\right) e_{2} \\
\bar{f}(\xi) & =\int_{0}^{\infty} t^{\left(s_{1} e_{1}+s_{2} e_{2}\right)-1}\left(f_{1}(t) e_{1}+f_{2}(t) e_{2}\right) d t \\
\bar{f}(\xi) & =\int_{0}^{\infty} t^{\xi-1} f(t) d t
\end{aligned}
$$

where $\xi=s_{1} e_{1}+s_{2} e_{2}$ and $\bar{f}(\xi)$ is analytic and convergent in the strip

$$
\begin{aligned}
\Omega= & \left\{\xi: \xi=s_{1} e_{1}+s_{2} e_{2} \in C_{2} ; \alpha<\operatorname{Re}\left(P_{1}: \xi\right)<\beta ; \alpha<\operatorname{Re}\left(P_{2}: \xi\right)<\beta ;\right. \\
& \left.\alpha=\max \left(\alpha_{1}, \alpha_{2}\right) \text { and } \beta=\min \left(\beta_{1}, \beta_{2}\right)\right\} .
\end{aligned}
$$


$\because \alpha<\operatorname{Re}\left(s_{1}\right)=x_{1}<\beta$ and $\alpha<\operatorname{Re}\left(s_{2}\right)=x_{2}<\beta$, we have

$$
\begin{aligned}
\xi= & \left(x_{1}+i_{1} y_{1}\right) e_{1}+\left(x_{2}+i_{1} y_{2}\right) e_{2}=\left(x_{1}+i_{1} y_{1}\right)\left(\frac{1+i_{1} i_{2}}{2}\right) \\
& +\left(x_{2}+i_{1} y_{2}\right)\left(\frac{1-i_{1} i_{2}}{2}\right) \\
= & \frac{x_{1}+x_{2}}{2}+\left(\frac{y_{1}+y_{2}}{2}\right) i_{1}+\left(\frac{y_{2}-y_{1}}{2}\right) i_{2}+\left(\frac{x_{1}-x_{2}}{2}\right) i_{1} i_{2} .
\end{aligned}
$$

Now, there are three possible cases:

1. If $x_{1}=x_{2}=a_{0}$ (say) then $\frac{x_{1}-x_{2}}{2}=0$ and $\frac{x_{1}+x_{2}}{2}=a_{0}$.

Hence, if $\xi=a_{0}+a_{1} i_{1}+a_{2} i_{2}{ }^{2}+a_{3} i_{1} i_{2}$, then $\alpha<a_{0}<\beta$ and $a_{3}=0$.

2. If $x_{1}>x_{2}$, then $\frac{x_{1}-x_{2}}{2}>0$,

$\frac{x_{1}+x_{2}}{2}<\frac{\beta+x_{2}}{2}<\frac{\beta+x_{2}}{2}+\frac{\beta-x_{1}}{2}=\beta-\frac{x_{1}-x_{2}}{2}$

and $\frac{x_{1}+x_{2}}{2}>\frac{\alpha+x_{1}}{2}>\frac{\alpha+x_{1}}{2}+\frac{\alpha-x_{2}}{2}=\alpha+\frac{x_{1}-x_{2}}{2}$.

Thus, $\alpha+a_{3}<a_{0}<\beta-a_{3}$ and $a_{3}>0$.

3. If $x_{1}<x_{2}$, then $\frac{x_{1}-x_{2}}{2}<0$,

$\frac{x_{1}+x_{2}}{2}<\frac{\beta+x_{1}}{2}<\frac{\beta+x_{1}}{2}+\frac{\beta-x_{2}}{2}=\beta+\frac{x_{1}-x_{2}}{2}$

and $\frac{x_{1}+x_{2}}{2}>\frac{\alpha+x_{2}}{2}>\frac{\alpha+x_{2}}{2}+\frac{\alpha-x_{1}}{2}=\alpha-\frac{x_{1}-x_{2}}{2}$.

Thus, $\alpha-a_{3}<a_{0}<\beta+a_{3}$ and $a_{3}<0$.

These three conditions can be written in the following set builder form

$$
\begin{aligned}
& \Omega_{1}=\left\{\xi=a_{0}+a_{1} i_{1}+a_{2} i_{2}+a_{3} i_{1} i_{2}: \alpha<a_{0}<\beta \text { and } a_{3}=0\right\}, \\
& \Omega_{2}=\left\{\xi=a_{0}+a_{1} i_{1}+a_{2} i_{2}+a_{3} i_{1} i_{2}: \alpha+a_{3}<a_{0}<\beta-a_{3} \text { and } a_{3}>0\right\}, \\
& \Omega_{3}=\left\{\xi=a_{0}+a_{1} i_{1}+a_{2} i_{2}+a_{3} i_{1} i_{2}: \alpha-a_{3}<a_{0}<\beta+a_{3} \text { and } a_{3}<0\right\} .
\end{aligned}
$$

Thus, $\alpha<\operatorname{Re}\left(P_{1}: \xi\right)<\beta$ and $\alpha<\operatorname{Re}\left(P_{2}: \xi\right)<\beta$ implies $\xi \in \Omega_{1} \cup \Omega_{2} \cup \Omega_{3}=\Omega$ which can be defined as:

$$
\Omega=\left\{\xi=a_{0}+a_{1} i_{1}+a_{2} i_{2}+a_{3} i_{1} i_{2} \in C_{2}: \alpha+\left|a_{3}\right|<a_{0}<\beta-\left|a_{3}\right|\right\}
$$

or equivalently,

$$
\Omega=\left\{\xi \in C_{2}: \alpha+\left|\operatorname{Im}_{j}(\xi)\right|<\operatorname{Re}(\xi)<\beta-\left|\operatorname{Im}_{j}(\xi)\right|\right\}
$$

where $\operatorname{Im}_{j}(\xi)$ denotes the imaginary part w.r.t. $j$ unit of a bicomplex number.

Conversely, the existence condition of bicomplex Mellin transform $\bar{f}(\xi)$ can be obtained in the following way:

$$
\begin{aligned}
& \text { If } \xi=a_{0}+a_{1} i_{1}+a_{2} i_{2}+a_{3} i_{1} i_{2} \in \Omega, \\
& \alpha+\left|a_{3}\right|<a_{0}<\beta-\left|a_{3}\right| .
\end{aligned}
$$

Now, in idempotent components, $\xi$ can be expressed as

$$
\begin{aligned}
\xi & =a_{0}+a_{1} i_{1}+a_{2} i_{2}+a_{3} i_{1} i_{2} \\
& =\left[\left(a_{0}+a_{3}\right)+i_{1}\left(a_{1}-a_{2}\right)\right] e_{1}+\left[\left(a_{0}-a_{3}\right)+i_{1}\left(a_{1}+a_{2}\right)\right] e_{2} \\
& =s_{1} e_{1}+s_{2} e_{2} .
\end{aligned}
$$

Depending on the value of $a_{3}$, there arises three cases:

1. $a_{3}=0$ and $\alpha<a_{0}<\beta$ which trivially leads $\alpha<a_{0}+a_{3}<\beta$ and $\alpha<a_{0}-a_{3}<\beta$. 
2. When $a_{3}>0$, from the inequality (2.8) $\alpha+a_{3}<a_{0}<\beta-a_{3}$, we get $\alpha<a_{0}-a_{3}$ and $a_{0}+a_{3}<\beta$. This result can be interpreted as $\alpha<a_{0}-a_{3}<a_{0}+a_{3}<\beta$.

3. When $a_{3}<0$, from the inequality (2.8) $\alpha-a_{3}<a_{0}<\beta+a_{3}$, we get $\alpha<a_{0}+a_{3}$ and $a_{0}-a_{3}<\beta$. This result can be interpreted as $\alpha<a_{0}+a_{3}<a_{0}-a_{3}<\beta$.

Hence the result.

Now, we define the Mellin transform in the bicomplex space as follows:

Definition 2.1. Let $f(t)$ be a bicomplex-valued continuous function on the interval $(0, \infty)$ with $f(t)=O\left(t^{-\alpha}\right)$ as $t \rightarrow 0^{+}$and $f(t)=O\left(t^{-\beta}\right)$ as $t \rightarrow \infty$, where $\alpha<\beta$. Then bicomplex Mellin transform of $f(t)$ defined as

$$
\mathfrak{M}[f(t) ; \xi]=\int_{0}^{\infty} t^{\xi-1} f(t) d t=\bar{f}(\xi), \quad \xi \in \Omega
$$

where $\bar{f}(\xi)$ is analytic and convergent in $\Omega$ defined in

$$
\Omega=\left\{\xi \in C_{2}: \alpha+\left|\operatorname{Im}_{j}(\xi)\right|<\operatorname{Re}(\xi)<\beta-\left|\operatorname{Im}_{j}(\xi)\right|\right\}
$$

where $\operatorname{Im}_{j}(\xi)$ denotes the imaginary part w.r.t. $j$ unit of a bicomplex number.

Following is the illustration to explain the process of finding the bicomplex Mellin transform of a bicomplex valued function.

Example 2.2. Let $f(t)=t^{a} U\left(t-t_{0}\right)$, where $U\left(t-t_{0}\right)$ is unit-step function, then

$$
\mathfrak{M}[f(t) ; \xi]=-\frac{t_{0}^{\xi+a}}{\xi+a}, \quad \operatorname{Re}(\xi+a)<-\left|\operatorname{Im}_{j}(\xi+a)\right| .
$$

Solution. By applying the definition of bicomplex Mellin transform

$$
\begin{aligned}
\mathfrak{M}[f(t) ; \xi] & =\int_{0}^{\infty} t^{\xi-1} t^{a} U\left(t-t_{0}\right) d t \\
& =\int_{t_{0}}^{\infty} t^{\xi+a-1} d t \\
& =-\frac{t_{0}^{\xi+a}}{\xi+a} .
\end{aligned}
$$

Table 1. Bicomplex Mellin transform of some basic functions

\begin{tabular}{|c|c|c|c|}
\hline S.No. & $f(t)$ & $\begin{array}{l}\text { Bicomplex } \\
\text { Hankel } \\
\text { Transform } \\
F(\xi)\end{array}$ & Region of Convergence \\
\hline 1. & $(1+t)^{-a}$ & $\frac{\Gamma(\xi) \Gamma(a-\xi)}{\Gamma(a)}$ & $\left|\operatorname{Im}_{j}(a-\xi)\right|<\operatorname{Re}(a-\xi)$ \\
\hline 2. & $(1+t)^{-1}$ & $\frac{\pi}{\sin (\pi \xi)}$ & $\left|\operatorname{Im}_{j}(\xi)\right|<\operatorname{Re}(\xi)<1-\left|\operatorname{Im}_{j}(\xi)\right|$ \\
\hline 3. & $e^{n t}, n>0$ & $\frac{\Gamma(\xi)}{n \xi}$ & $\operatorname{Re}(\xi)>\left|\operatorname{Im}_{j}(\xi)\right|$ \\
\hline 4. & $\sin (a t), a>0$ & $\frac{\Gamma(\xi) \sin \left(\frac{\pi \xi}{2}\right)}{a^{\xi}}$ & $-1+\left|\operatorname{Im}_{j}(\xi)\right|<\operatorname{Re}(\xi)<1-\left|\operatorname{Im}_{j}(\xi)\right|$ \\
\hline 5. & $\cos (a t), a>0$ & $\frac{\Gamma(\xi) \cos \left(\frac{\pi \xi}{2}\right)}{\frac{a \xi}{\pi}}$ & $\left|\operatorname{Im}_{j}(\xi)\right|<\operatorname{Re}(\xi)<1-\left|\operatorname{Im}_{j}(\xi)\right|$ \\
\hline 6. & $\log (1+t)$ & $\frac{\frac{\pi}{\xi \sin (\pi \xi)}}{\xi-a}$ & $-1+\left|\operatorname{Im}_{j}(\xi)\right|<\operatorname{Re}(\xi)<-\left|\operatorname{Im}_{j}(\xi)\right|$ \\
\hline 7. & $t^{-a}$ & $\frac{1}{\operatorname{Re}(\xi-a)<-\left|\operatorname{Im}_{j}(\xi-a)\right|}$ \\
\hline
\end{tabular}




\section{Properties of bicomplex Mellin transform}

In this section, we discuss the basic properties of bicomplex Mellin transform viz. linearity property, change of scale property, shifting property, Mellin transform of derivatives and operators, relation with bicomplex Laplace transform and some other properties.

Theorem 3.1. (Linearity Property). Let $f(t)$ and $g(t)$ are bicomplex-valued functions with $f(t)=O\left(t^{-\alpha_{1}}\right), g(t)=O\left(t^{-\alpha_{2}}\right)$ as $t \rightarrow 0^{+}$and $f(t)=O\left(t^{-\beta_{1}}\right), g(t)=$ $O\left(t^{-\beta_{2}}\right)$ as $t \rightarrow \infty$, with $\max \left(\alpha_{1}, \alpha_{2}\right)+\left|\operatorname{Im}_{j}(\xi)\right|<\operatorname{Re}(\xi)<\min \left(\beta_{1}, \beta_{2}\right)-\left|\operatorname{Im}_{j}(\xi)\right|$, then

$$
\mathfrak{M}\left[c_{1} f(t)+c_{2} g(t) ; \xi\right]=c_{1} \mathfrak{M}[f(t) ; \xi]+c_{2} \mathfrak{M}[g(t) ; \xi]
$$

where $c_{1}$ and $c_{2}$ are arbitrary constants.

Proof. By applying the definition of bicomplex Mellin transform

$$
\begin{aligned}
\mathfrak{M}\left[c_{1} f(t)+c_{2} g(t) ; \xi\right] & =\int_{0}^{\infty} t^{\xi-1}\left[c_{1} f(t)+c_{2} g(t)\right] d t \\
& =c_{1} \int_{0}^{\infty} t^{\xi-1} f(t) d t+c_{2} \int_{0}^{\infty} t^{\xi-1} g(t) d t \\
& =c_{1} \mathfrak{M}[f(t) ; \xi]+c_{2} \mathfrak{M}[g(t) ; \xi] .
\end{aligned}
$$

Theorem 3.2. (Change of scale property). Let $\bar{f}(\xi)$ be the bicomplex Mellin transform of bicomplex-valued function $f(t)$, then

$$
\mathfrak{M}[f(a t) ; \xi]=a^{-\xi} \bar{f}(\xi), \quad \xi \in \Omega, a>0
$$

where $\Omega$ is defined in (2.9).

Proof. By applying the definition of bicomplex Mellin transform

$$
\begin{aligned}
\mathfrak{M}[f(a t) ; \xi] & =\int_{0}^{\infty} t^{\xi-1} f(a t) d t, \quad\left[\text { where } \xi=s_{1} e_{1}+s_{2} e_{2}\right] \\
& =\left(\int_{0}^{\infty} t^{s_{1}-1} f_{1}(a t) d t\right) e_{1}+\left(\int_{0}^{\infty} t^{s_{2}-1} f_{2}(a t) d t\right) e_{2}
\end{aligned}
$$

Put $a t=u$, to obtain

$$
\begin{aligned}
& =\frac{1}{a^{s_{1}}}\left(\int_{0}^{\infty} t^{s_{1}-1} f_{1}(u) d t\right) e_{1}+\frac{1}{a^{s_{2}}}\left(\int_{0}^{\infty} t^{s_{2}-1} f_{2}(u) d t\right) e_{2} \\
& =\frac{1}{a^{s_{1} e_{1}+s_{2} e_{2}}} \int_{0}^{\infty} t^{s_{1} e_{1}+s_{2} e_{2}-1}\left(f_{1}(u) e_{1}+f_{2}(u) e_{2}\right) d t \\
& =\frac{1}{a^{\xi}} \int_{0}^{\infty} t^{\xi-1} f(u) d t \\
& =\frac{\bar{f}(\xi)}{a^{\xi}} .
\end{aligned}
$$


Theorem 3.3. (Bicomplex Mellin Transform of Derivatives). Let $\bar{f}(\xi)$ be bicomplex Mellin transform of bicomplex-valued function $f(t)$, then

$$
\mathfrak{M}\left[f^{(n)}(t) ; \xi\right]=(-1)^{n} \frac{\Gamma(\xi)}{\Gamma(\xi-n)} \bar{f}(\xi-n), \quad(\xi-n) \in \Omega
$$

where $\Omega$ is defined in (2.9) and provided $t^{\xi-r-1} f^{(r)}(t)$ vanishes as $t \rightarrow 0$ and as $t \rightarrow \infty$ for $r=0,1,2, \cdots,(n-1)$.

Proof. For $n=1$, according to the definition of bicomplex Mellin transform,

$$
\mathfrak{M}\left[f^{\prime}(t) ; \xi\right]=\int_{0}^{\infty} t^{\xi-1} f^{\prime}(t) d t
$$

which on integration by parts, gives

$$
\begin{aligned}
\mathfrak{M}\left[f^{\prime}(t) ; \xi\right] & =\left.t^{\xi-1} f(t)\right|_{0} ^{\infty}-(\xi-1) \int_{0}^{\infty} t^{\xi-2} f(t) d t \\
& =-(\xi-1) \bar{f}(\xi-1) .
\end{aligned}
$$

Therefore, the result is true for $n=1$. Let the the above result is true for $n=m$

$$
\mathfrak{M}\left[f^{(m)}(t) ; \xi\right]=(-1)^{m} \frac{\Gamma(\xi)}{\Gamma(\xi-m)} \bar{f}(\xi-m) .
$$

Now, for $n=m+1$

$$
\mathfrak{M}\left[f^{(m+1)}(t) ; \xi\right]=\int_{0}^{\infty} t^{\xi-1} f^{(m+1)}(t) d t
$$

Integrating by parts, we get

$$
\begin{aligned}
\mathfrak{M}\left[f^{(m+1)}(t) ; \xi\right] & =\left.t^{\xi-1} f^{(m)}(t)\right|_{0} ^{\infty}-(\xi-1) \int_{0}^{\infty} t^{\xi-2} f^{(m)}(t) d t \\
& =-(\xi-1)(-1)^{m} \frac{\Gamma(\xi-1)}{\Gamma(\xi-m-1)} \bar{f}(\xi-m-1),[\operatorname{using}(3.4)] \\
& =(-1)^{m+1} \frac{\Gamma(\xi)}{\Gamma(\xi-m-1)} \bar{f}(\xi-m-1) .
\end{aligned}
$$

Therefore, the result is true for $n=m+1$. Hence, by the principal of mathematical induction the result is true for all $n=1,2, \cdots$. Therefore,

$$
\mathfrak{M}\left[f^{(n)}(t) ; \xi\right]=(-1)^{n} \frac{\Gamma(\xi)}{\Gamma(\xi-n)} \bar{f}(\xi-n) .
$$

Theorem 3.4. (Shifting Property). Let $\bar{f}(\xi)$ be bicomplex Mellin transform of bicomplex-valued function $f(t)$. Then

$$
\mathfrak{M}\left[t^{a} f(t) ; \xi\right]=\bar{f}(\xi+a), \quad(\xi+a) \in \Omega, a \in C_{2}
$$

where $\Omega$ is defined in (2.9).

Proof. By applying the definition of bicomplex Mellin transform,

$$
\mathfrak{M}\left[t^{a} f(t) ; \xi\right]=\int_{0}^{\infty} t^{\xi-1} t^{a} f(t) d t=\int_{0}^{\infty} t^{\xi+a-1} f(t) d t=\bar{f}(\xi+a) .
$$


Theorem 3.5. Let $\bar{f}(\xi)$ be bicomplex Mellin transform of bicomplex-valued function $f(t)$. Then

$$
\mathfrak{M}\left[f\left(t^{a}\right) ; \xi\right]=\frac{1}{a} \bar{f}\left(\frac{\xi}{a}\right), \quad \frac{\xi}{a} \in \Omega, 0 \neq a \in C_{0}
$$

where $\Omega$ is defined in (2.9).

Proof. By applying the definition of bicomplex Mellin transform,

$$
\begin{aligned}
\mathfrak{M}\left[f\left(t^{a}\right) ; \xi\right] & =\int_{0}^{\infty} t^{\xi-1} f\left(t^{a}\right) d t \\
& \left.=\frac{1}{a} \int_{0}^{\infty} u^{\frac{\xi}{a}-1} f(u) d u \quad \text { [substituting } t^{a}=u\right] \\
& =\frac{1}{a} \bar{f}\left(\frac{\xi}{a}\right) .
\end{aligned}
$$

Theorem 3.6. Let $\bar{f}(\xi)$ be bicomplex Mellin transform of bicomplex-valued function $f(t)$. Then

$$
\mathfrak{M}\left[t^{n} f^{(n)}(t) ; \xi\right]=(-1)^{n} \frac{\Gamma(\xi+n)}{\Gamma(\xi)} \bar{f}(\xi), \quad \xi \in \Omega
$$

where $\Omega$ is defined in (2.9) and provided $t^{\xi-r} f^{(r)}(\xi)$ vanishes as $t \rightarrow 0$ and as $t \rightarrow \infty$ for $r=0,1,2, \cdots,(n-1)$.

Proof. By applying the definition of bicomplex Mellin transform,

$$
\begin{aligned}
\mathfrak{M}\left[t^{n} f^{(n)}(t) ; \xi\right] & =\int_{0}^{\infty} t^{\xi-1} t^{n} f^{(n)}(t) d t, \quad\left[\text { where } \xi=s_{1} e_{1}+s_{2} e_{2}\right] \\
& =\left(\int_{0}^{\infty} t^{s_{1}-1} t^{n} f_{1}^{(n)}(t) d t\right) e_{1}+\left(\int_{0}^{\infty} t^{s_{2}-1} t^{n} f_{2}^{(n)}(t) d t\right) e_{2} \\
& =(-1)^{n} \frac{\Gamma\left(s_{1}+n\right)}{\Gamma\left(s_{1}\right)} \bar{f}_{1}\left(s_{1}\right) e_{1}+(-1)^{n} \frac{\Gamma\left(s_{2}+n\right)}{\Gamma\left(s_{2}\right)} \bar{f}_{2}\left(s_{2}\right) e_{2}, \\
& =(-1)^{n} \frac{\Gamma\left(s_{1} e_{1}+s_{2} e_{2}+n\right)}{\Gamma\left(s_{1} e_{1}+s_{2} e_{2}\right)}\left(\bar{f}_{1}\left(s_{1}\right) e_{1}+\bar{f}_{2}\left(s_{2}\right) e_{2}\right) \\
& =(-1)^{n} \frac{\Gamma(\xi+n)}{\Gamma(\xi)} \bar{f}(\xi) .
\end{aligned}
$$

Theorem 3.7. (Bicomplex Mellin Transform of Differential Operators). Let $\bar{f}(\xi)$ be bicomplex Mellin transform of bicomplex-valued function $f(t)$. Then

$$
\mathfrak{M}\left[\left(t \frac{d}{d t}\right)^{2} f(t) ; \xi\right]=\mathfrak{M}\left[t^{2} f^{\prime \prime}(t)+t f^{\prime}(t) ; \xi\right]=(-1)^{2} \xi^{2} \bar{f}(\xi), \xi \in \Omega
$$

where $\Omega$ is defined in (2.9). 
Proof. By applying the definition of bicomplex Mellin transform,

$$
\begin{aligned}
\mathfrak{M}\left[\left(t \frac{d}{d t}\right)^{2} f(t) ; \xi\right] & =\mathfrak{M}\left[t^{2} f^{\prime \prime}(t)+t f^{\prime}(t) ; \xi\right] \\
& =\mathfrak{M}\left[t^{2} f^{\prime \prime}(t) ; \xi\right]+\mathfrak{M}\left[t f^{\prime}(t) ; \xi\right] \\
& =\xi(\xi+1) \bar{f}(\xi)-\xi \bar{f}(\xi) \\
& =(-1)^{2} \xi^{2} \bar{f}(\xi) .
\end{aligned}
$$

In general,

$$
\mathfrak{M}\left[\left(t \frac{d}{d t}\right)^{n} f(t) ; \xi\right]=(-1)^{n} \xi^{n} \bar{f}(\xi) .
$$

Theorem 3.8. (Bicomplex Mellin Transform of Integrals). Let $\bar{f}(\xi)$ be bicomplex Mellin transform of bicomplex-valued function $f(t)$. Then

$$
\mathfrak{M}\left[\int_{0}^{t} f(x) d x ; \xi\right]=-\frac{1}{\xi} \bar{f}(\xi+1), \quad(\xi+1) \in \Omega
$$

where $\Omega$ is defined in (2.9).

Proof. We write

$$
g(t)=\int_{0}^{t} f(x) d x
$$

so that $g^{\prime}(t)=f(t)$ with $g(0)=0$. Taking the bicomplex Mellin transform of $g^{\prime}(t)$ and using Theorem 3.3 therein, we get

$$
\begin{aligned}
\mathfrak{M}\left[g^{\prime}(t) ; \xi\right] & =-(\xi-1) \mathfrak{M}[g(t) ; \xi-1] \\
& =-(\xi-1) \mathfrak{M}\left[\int_{0}^{t} f(x) d x ; \xi-1\right]
\end{aligned}
$$

Replacing $\xi$ by $\xi+1$, we get the desired result (3.9).

\subsection{Relation with Bicomplex Laplace Transform}

The bicomplex Laplace transform and its properties are discussed by Kumar A. and Kumar P. [14]. It is defined as

Definition 3.9. Let $f(t)$ be a bicomplex-valued function of exponential order $\alpha \in C_{0}$. Then Laplace Transform of $f(t)$ for $t \geq 0$ can be defined as:

$$
L\{f(t)\}=\int_{0}^{\infty} f(t) e^{-\xi t} d t=F(\xi)
$$

Here $F(\xi)$ exist and is convergent for all $\xi \in D=D_{1} \cup D_{2} \cup D_{3}$ or

$$
D=\left\{\xi \in C_{2}: H_{\rho}(\xi) \text { represent a Right half-plane } a_{0}>\alpha+\left|a_{3}\right|\right\}
$$


where

$$
\begin{aligned}
& D_{1}=\left\{\xi=a_{0}+a_{1} i_{1}+a_{2} i_{2}+a_{3} i_{1} i_{2}: a_{0}>\alpha, a_{3}=0\right\} \\
& D_{2}=\left\{\xi=a_{0}+a_{1} i_{1}+a_{2} i_{2}+a_{3} i_{1} i_{2}: a_{0}>\alpha+a_{3}, a_{3}>0\right\}
\end{aligned}
$$

and

$$
D_{3}=\left\{\xi=a_{0}+a_{1} i_{1}+a_{2} i_{2}+a_{3} i_{1} i_{2}: a_{0}>\alpha-a_{3}, a_{3}<0\right\} .
$$

In $D$, there are infinite $\xi$ which have same $H_{\rho}$ hyperbolic projection because $a_{1}$ and $a_{2}$ are free from restriction.

Therefore, the usual right-sided bicomplex Laplace transform is analytic in halfplane $\operatorname{Re}(\xi)>\alpha+\left|\operatorname{Im}_{j}(\xi)\right|$. In the same way, left-sided bicomplex Laplace transform is analytic in the region $\operatorname{Re}(\xi)<\beta-\left|\operatorname{Im}_{j}(\xi)\right|$. If the two half-planes overlap, the region of analyticity of the two-sided bicomplex Laplace transform is thus the strip

$$
D=\left\{\xi \in C_{2}: \alpha+\left|\operatorname{Im}_{j}(\xi)\right|<\operatorname{Re}(\xi)<\beta-\left|\operatorname{Im}_{j}(\xi)\right|\right\} .
$$

Hence, $D$ is equivalent to $\Omega$ defined in $(2.9)$.

Theorem 3.10. Let $\bar{f}(\xi)$ be bicomplex Mellin transform of bicomplex-valued function $f(t)$. Then

$$
\mathfrak{M}[f(t) ; \xi]=\int_{-\infty}^{\infty} e^{\xi x} f\left(e^{-x}\right) d x=L\left[f\left(e^{-x}\right) ; \xi\right], \quad \xi \in \Omega
$$

where $\Omega$ is defined in (2.9).

Proof. Taking $t=e^{-x}$ in the definition of bicomplex Mellin transform

$$
\mathfrak{M}[f(t) ; \xi]=\int_{0}^{\infty} t^{\xi-1} f(t) d t
$$

we get

$$
\mathfrak{M}[f(t) ; \xi]=\int_{-\infty}^{\infty} e^{\xi x} f\left(e^{-x}\right) d x=L\left[f\left(e^{-x}\right) ; \xi\right]
$$

\section{Inversion of bicomplex Mellin transform}

In this section, we discuss the inversion of bicomplex Mellin transform. Let $\bar{f}(\xi)$ be the bicomplex Mellin transform of bicomplex-valued continuous function $f(t)$. Then $\bar{f}(\xi)=\bar{f}_{1}\left(s_{1}\right) e_{1}+\bar{f}_{2}\left(s_{2}\right) e_{2}$ is analytic in the strip $\Omega$, which is defined in (2.6). The inverse formula for complex mellin transform (see, e.g. Poularikas A.D. [15, chapter 11] and Davies B. [6, p. 195-210]) is

$$
\begin{aligned}
f_{1}(t) & =\frac{1}{2 \pi i_{1}} \int_{c_{1}-i_{1} \infty}^{c_{1}+i_{1} \infty} t^{-s_{1}} \bar{f}_{1}\left(s_{1}\right) d s_{1}, \quad \alpha_{1}<c_{1}<\beta_{1} \\
& =\frac{1}{2 \pi i_{1}} \int_{\Omega_{1}} t^{-s_{1}} \bar{f}_{1}\left(s_{1}\right) d s_{1}
\end{aligned}
$$


where, $\Omega_{1}$ is defined in (2.2). Similarly, another inverse formula for complex Mellin transform is

$$
\begin{aligned}
f_{2}(t) & =\frac{1}{2 \pi i_{1}} \int_{c_{2}-i_{1} \infty}^{c_{2}+i_{1} \infty} t^{-s_{2}} \bar{f}_{2}\left(s_{2}\right) d s_{2}, \quad \alpha_{2}<c_{2}<\beta_{2} \\
& =\frac{1}{2 \pi i_{1}} \int_{\Omega_{2}} t^{-s_{2}} \bar{f}_{2}\left(s_{2}\right) d s_{2}
\end{aligned}
$$

where, $\Omega_{2}$ is defined in $(2.3)$.

Now, using complex inversions (4.1) and (4.2), we obtain the bicomplex-valued function as

$$
\begin{aligned}
f(t) & =f_{1}(t) e_{1}+f_{2}(t) e_{2} \\
& =\left(\frac{1}{2 \pi i_{1}} \int_{\Omega_{1}} t^{-s_{1}} \bar{f}_{1}\left(s_{1}\right) d s_{1}\right) e_{1}+\left(\frac{1}{2 \pi i_{1}} \int_{\Omega_{2}} t^{-s_{2}} \bar{f}_{2}\left(s_{2}\right) d s_{2}\right) e_{2} \\
& =\frac{1}{2 \pi i_{1}}\left(\int_{\left(\Omega_{1}, \Omega_{2}\right)} t^{-\left(s_{1} e_{1}+s_{2} e_{2}\right)}\left(\bar{f}_{1}\left(s_{1}\right) e_{1}+\bar{f}_{2}\left(s_{2}\right) e_{2}\right) d\left(s_{1} e_{1}+s_{2} e_{2}\right)\right) \\
& =\frac{1}{2 \pi i_{1}} \int_{\Omega} t^{-s_{1}} \bar{f}(\xi) d \xi
\end{aligned}
$$

where, $\Omega$ is defined in (2.9).

Consider the problem of asymptotically expanding $f(t)$ as $t \rightarrow 0^{+}$, when $\bar{f}(\xi)$ is known to be continuable in $-M+\left|\operatorname{Im}_{j}(\xi)\right| \leq \operatorname{Re}(\xi) \leq \alpha-\left|\operatorname{Im}_{j}(\xi)\right|$ for some $M>0$. We also postulate that $\bar{f}(\xi)$ has finitely many poles $\lambda_{k}$ such that $\operatorname{Re}\left(\lambda_{k}\right)>$ $-M+\left|\operatorname{Im}_{j}\left(\lambda_{k}\right)\right|$. Then

$$
f(t)=\sum_{\lambda_{k} \in \mathcal{K}} \operatorname{Res}\left[t^{-\xi} \bar{f}(\xi), \xi=\lambda_{k}\right]+O\left(t^{M}\right), \quad \text { as } t \rightarrow 0^{+}
$$

where $\mathcal{K}$ is the set of singularities and $M$ is as large as we want. Similarly, for problem of asymptotically expanding $f(t)$ as $t \rightarrow \infty$. Then contour taken in right and side of the fundamental strip, we have

$$
f(t)=-\sum_{\lambda_{k} \in \mathcal{K}} \operatorname{Res}\left[t^{-\xi} \bar{f}(\xi), \xi=\lambda_{k}\right]+O\left(t^{-M}\right), \quad \text { as } t \rightarrow \infty .
$$

Following is the illustration to explain the process of finding the inverse bicomplex Mellin transform.

Example 4.1. Let $\bar{f}(\xi)=\frac{1}{(\xi-a)(\xi-b)}$, for $\operatorname{Re}(\xi-a)<-\left|\operatorname{Im}_{j}(\xi-a)\right|$ and $\operatorname{Re}(a-b)<$ $-\left|\operatorname{Im}_{j}(a-b)\right|$. Then find the inverse bicomplex Mellin transform $f(t)$ of $\bar{f}(\xi)$. 
Solution. By applying the inverse bicomplex Mellin transform on $\bar{f}(\xi)$

$$
\begin{aligned}
f(t) & =\frac{1}{2 \pi i_{1}} \int_{\Omega} t^{-\xi} \bar{f}(\xi) d \xi \\
& =-\left[\operatorname{Res}\left(t^{-\xi} \frac{1}{(\xi-a)(\xi-b)}, \xi=a\right)+\operatorname{Res}\left(t^{-\xi} \frac{1}{(\xi-a)(\xi-b)}, \xi=b\right)\right] \\
& =\frac{1}{b-a}\left(t^{-a}-t^{-b}\right) .
\end{aligned}
$$

\section{Application of bicomplex Mellin transform}

In this paper, we are interested in determining the extent to which the output voltage $V$ and current $I$ using by bicomplex concept differs from their input values as the length of the transmission line tends to a very small value.

Now, let us define bicomplex scalar field as

$$
F \equiv V+i_{2} I
$$

where voltage $V$ and current $I$ are complex scalar fields. Now, we consider an equivalent circuit of a transmission line of small length $\Delta x$ containing resistance $R \Delta x$, capacitance $C \Delta x$, and inductance $L \Delta x$ as shown in Figure 1.

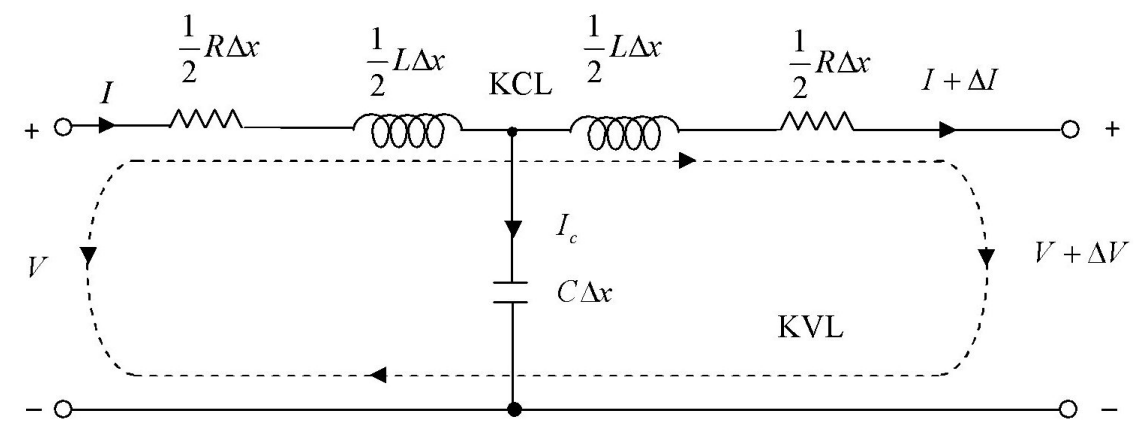

Figure 1. Equivalent circuit of a transmission line

The above figure is a symmetrical network. By using the Kirchhoff's voltage law (KVL), we have

$$
V=\frac{1}{2} R I \Delta x+\frac{1}{2} L \frac{\partial I}{\partial t} \Delta x+\frac{1}{2} L \frac{\partial}{\partial t}(I+\Delta I) \Delta x+\frac{1}{2} R(I+\Delta I) \Delta x+V+\Delta V .
$$

Dividing (5.2) by $\Delta x$ and simplifying, we get

$$
\frac{\Delta V}{\Delta x}=-\left[R I+L \frac{\partial I}{\partial t}+\left(\frac{L}{2} \frac{\partial}{\partial t} \frac{\Delta I}{\Delta x}+\frac{R}{2} \frac{\Delta I}{\Delta x}\right) \Delta x\right] .
$$

Taking limit as $\Delta x \rightarrow 0$, we get

$$
\frac{\partial V}{\partial x}=-\left[R I+L \frac{\partial I}{\partial t}\right]
$$


By applying Kirchhoff's current law (KCL) on the equivalent circuit of the transmission line, we get

$$
\begin{aligned}
I & =I_{c}+I+\Delta I \\
& =C \frac{\partial}{\partial t}\left(V+\frac{\Delta V}{2}\right) \Delta x+I+\Delta I .
\end{aligned}
$$

Dividing (5.5) by $\Delta x$ and simplifying, we get

$$
\frac{\Delta I}{\Delta x}=-\left[C \frac{\partial V}{\partial t}+\frac{C}{2} \frac{\partial}{\partial t}\left(\frac{\Delta V}{\Delta x}\right) \Delta x\right] .
$$

Taking limit as $\Delta x \rightarrow 0$, we get

$$
\frac{\partial I}{\partial x}=-C \frac{\partial V}{\partial t}
$$

The differential equations in (5.4) and (5.7) describes the evaluation of current and voltage in a lossy transmission line. Differentiating (5.4) w.r.t. $x$ and simplifying using (5.7), we get

$$
\frac{\partial^{2} V}{\partial x^{2}}=C L \frac{\partial^{2} V}{\partial t^{2}}+C R \frac{\partial V}{\partial t} .
$$

Similarly, differentiating (5.7) w.r.t. $x$ and simplifying using (5.4), we get

$$
\frac{\partial^{2} I}{\partial x^{2}}=C L \frac{\partial^{2} I}{\partial t^{2}}+C R \frac{\partial I}{\partial t}
$$

Equations (5.8) and (5.9) are hyperbolic partial differential equations which describes the voltage and current along power transmission lines.

Combining equation (5.8) and (5.9) with the help of bicomplex unit $i_{2}$ as

$$
\begin{aligned}
\frac{\partial^{2} V}{\partial x^{2}}+i_{2} \frac{\partial^{2} I}{\partial x^{2}} & =C L\left(\frac{\partial^{2} V}{\partial t^{2}}+i_{2} \frac{\partial^{2} I}{\partial t^{2}}\right)+C R\left(\frac{\partial V}{\partial t}+i_{2} \frac{\partial I}{\partial t}\right) \\
\Rightarrow \quad \frac{\partial^{2}}{\partial x^{2}}\left(V+i_{2} I\right) & =C L \frac{\partial^{2}}{\partial t^{2}}\left(V+i_{2} I\right)+C R \frac{\partial}{\partial t}\left(V+i_{2} I\right) \\
\Rightarrow \quad \frac{\partial^{2}}{\partial x^{2}} F(x, t) & =C L \frac{\partial^{2}}{\partial t^{2}} F(x, t)+C R \frac{\partial}{\partial t} F(x, t)
\end{aligned}
$$

where $F(x, t)$ is bicomplex-valued function defined by (5.1).

In particular, a circuit which has resistance $R=\frac{1}{t}$, capacitance $C=t^{2}$ and inductance $L=1$. The differential equation (5.10) of bicomplex-valued function becomes

$$
\frac{\partial^{2}}{\partial x^{2}} F(x, t)=t^{2} \frac{\partial^{2}}{\partial t^{2}} F(x, t)+t \frac{\partial}{\partial t} F(x, t) .
$$

For finding the solution of partial differential equation (5.11), we assume boundary conditions as

$$
F(0, t)=0 \quad \text { and } \quad F(1, t)=A\left(\frac{1}{t^{a}}+\frac{1}{t^{b}}\right)
$$


where $A \in C_{2}, \operatorname{Re}(b-a)>\left|\operatorname{Im}_{j}(b-a)\right|$. By taking the bicomplex Mellin transform of (5.11) w.r.t. $t$ and making use of Theorem 3.7, we get

$$
\frac{d^{2}}{d x^{2}} \bar{F}(x, \xi)=\xi^{2} \bar{F}(x, \xi) .
$$

Therefore, by taking the bicomplex Mellin transform of (5.12) and using in solution of $(5.13)$, we get

$$
\bar{F}(x, \xi)=A\left[\frac{(-2 \xi+a+b)\left(e^{\xi x}-e^{-\xi x}\right)}{(\xi-a)(\xi-b)\left(e^{\xi}-e^{-\xi}\right)}\right]
$$

By taking the inverse bicomplex Mellin transform (5.14), we get

$$
F(x, t)=\frac{1}{2 \pi i_{1}} \int_{\Omega} t^{-\xi} \bar{F}(x, \xi) d \xi
$$

where $\bar{F}(x, \xi)$ is analytic in $\operatorname{Re}(\xi-a)>\left|\operatorname{Im}_{j}(\xi-a)\right|$. Then taking a semi-circle on the right-hand side of a large radius and using by residue theorem, we have

$$
\begin{aligned}
F(x, t)= & A\left[\frac{\sinh (a x)}{\sinh (a)} t^{-a}+\frac{\sinh (b x)}{\sinh (b)} t^{-b}\right] \\
= & A_{1}\left[\frac{\sinh \left(a_{1} x\right)}{\sinh \left(a_{1}\right)} t^{-a_{1}}+\frac{\sinh \left(b_{1} x\right)}{\sinh \left(b_{1}\right)} t^{-b_{1}}\right] e_{1} \\
& +A_{2}\left[\frac{\sinh \left(a_{2} x\right)}{\sinh \left(a_{2}\right)} t^{-a_{2}}+\frac{\sinh \left(b_{2} x\right)}{\sinh \left(b_{2}\right)} t^{-b_{2}}\right] e_{2}
\end{aligned}
$$

where $A=A_{1} e_{1}+A_{2} e_{2}, a=a_{1} e_{1}+a_{2} e_{2}$ and $b=b_{1} e_{1}+b_{2} e_{2}$. Therefore,

$$
\begin{aligned}
F(x, t) \equiv & V+i_{2} I \\
= & \frac{1}{2}\left\{A_{1}\left[\frac{\sinh \left(a_{1} x\right)}{\sinh \left(a_{1}\right)} t^{-a_{1}}+\frac{\sinh \left(b_{1} x\right)}{\sinh \left(b_{1}\right)} t^{-b_{1}}\right]\right. \\
& \left.+A_{2}\left[\frac{\sinh \left(a_{2} x\right)}{\sinh \left(a_{2}\right)} t^{-a_{2}}+\frac{\sinh \left(b_{2} x\right)}{\sinh \left(b_{2}\right)} t^{-b_{2}}\right]\right\} \\
& +i_{2} \frac{i_{1}}{2}\left\{A_{1}\left[\frac{\sinh \left(a_{1} x\right)}{\sinh \left(a_{1}\right)} t^{-a_{1}}+\frac{\sinh \left(b_{1} x\right)}{\sinh \left(b_{1}\right)} t^{-b_{1}}\right]\right. \\
& \left.-A_{2}\left[\frac{\sinh \left(a_{2} x\right)}{\sinh \left(a_{2}\right)} t^{-a_{2}}+\frac{\sinh \left(b_{2} x\right)}{\sinh \left(b_{2}\right)} t^{-b_{2}}\right]\right\} .
\end{aligned}
$$

Separating the bi-real and bi-imaginary parts of (5.16), we obtain the voltage and current of above model as

$$
\begin{aligned}
V(x, t)= & \frac{1}{2}\left\{A_{1}\left[\frac{\sinh \left(a_{1} x\right)}{\sinh \left(a_{1}\right)} t^{-a_{1}}+\frac{\sinh \left(b_{1} x\right)}{\sinh \left(b_{1}\right)} t^{-b_{1}}\right]\right. \\
& \left.+A_{2}\left[\frac{\sinh \left(a_{2} x\right)}{\sinh \left(a_{2}\right)} t^{-a_{2}}+\frac{\sinh \left(b_{2} x\right)}{\sinh \left(b_{2}\right)} t^{-b_{2}}\right]\right\}
\end{aligned}
$$


and

$$
\begin{aligned}
I(x, t)= & \frac{i_{1}}{2}\left\{A_{1}\left[\frac{\sinh \left(a_{1} x\right)}{\sinh \left(a_{1}\right)} t^{-a_{1}}+\frac{\sinh \left(b_{1} x\right)}{\sinh \left(b_{1}\right)} t^{-b_{1}}\right]\right. \\
& \left.-A_{2}\left[\frac{\sinh \left(a_{2} x\right)}{\sinh \left(a_{2}\right)} t^{-a_{2}}+\frac{\sinh \left(b_{2} x\right)}{\sinh \left(b_{2}\right)} t^{-b_{2}}\right]\right\} .
\end{aligned}
$$

\section{Conclusion}

The concept of bicomplex numbers has been applied for finding the solution of differential equations of bicomplex-valued function generated by network diagram. In this paper, we derive Mellin transform and its inverse in bicomplex space which is the generalization of complex Mellin transform. The application has been illustrated to find the solution of partial differential equation of bicomplex-valued function generated by a network. The bicomplex analysis has great advantage that it separates the voltage and current as complex components.

\section{References}

[1] Agarwal, R., Goswami, M.P., Agarwal, R.P., Bicomplex version of Stieltjes transform and applications, Dynamics of Continuous, Discrete and Impulsive Systems Series B: Applications \& Algorithms, 21(2014), no. 4-5, 229-246.

[2] Agarwal, R., Goswami, M.P., Agarwal, R.P., Convolution theorem and applications of bicomplex Laplace transform, Advances in Mathematical Sciences and Applications, 24(2014), no. 1, 113-127.

[3] Agarwal, R., Goswami, M.P., Agarwal, R.P., Tauberian theorem and applications of bicomplex Laplace-Stieltjes transform, Dynamics of Continuous, Discrete and Impulsive Systems, Series B: Applications \& Algorithms, 22(2015), 141-153.

[4] Agarwal, R., Goswami, M.P., Agarwal, R.P., Bochner theorem and applications of bicomplex Fourier-Stieltjes transform, Advanced Studies in Contemporary Mathematics, 26(2016), no. 2, 355-369.

[5] Banerjee, A., Datta, S.K., Hoque, A., Fourier transform and its inverse for functions of bicomplex variables, arXiv.org math arXiv:1404.4236, 2014, 1-20.

[6] Davies, B., Integral transforms and their applications, Springer, 2001.

[7] Debnath Loknath, Bhatta Dambaru, Integral transforms and their applications, Chaman \& Hall/CRC Taylor \& Francis Group, 2006.

[8] Dragoni, G.S., Sulle funzioni olomorfe di una variable bicomplessa, Reale Acad. d'Italia Mem. Class Sci. Fic. Mat. Nat., 5(1934), 597-665.

[9] Flajolet, P., Gourdon, X., Dumas, P., Mellin transforms and asymptotics: Harmonic sums, Theoretical Computer Science, 144(1995), 3-58.

[10] Futagawa, M., On the theory of functions of quaternary variable - I, Tohoku Math. J., 29(1928), 175-222.

[11] Futagawa, M., On the theory of functions of quaternary variable - II, Tohoku Math. J., 35(1932), 69-120. 
[12] Gerardi, F.R., Application of Mellin and Hankel transforms to networks with timevarying parameters, IRE Transaction on Circuit Theory, 6(1959), no. 2, 197-208.

[13] Goyal, R., Bicomplex polygamma function, Tokyo Journal of Mathematics, 30(2007), no. 2, 523-530.

[14] Kumar, A., Kumar, P., Bicomplex version of Laplace transform, International Journal of Engg. and Tech., 3(2011), no. 3, 225-232.

[15] Poularikas, A.D., The transforms and applications handbook, CRC Press, 1999.

[16] Price, G.B., An introduction to multicomplex spaces and functions, Marcel Dekker Inc., New York, 1991.

[17] Rönn, S., Bicomplex algebra and function theory, arXiv:math/0101200v1 [math.CV], (2001), 1-71.

[18] Segre, C., Le rappresentazioni reale delle forme complesse e gli enti iperalgebrici, Math. Ann., 40(1892), 413-467.

Ritu Agarwal

Department of Mathematics

Malaviya National Institute of Technology

Jaipur-302017, India

e-mail: ragarwal.maths@mnit.ac .in

Mahesh Puri Goswami

Department of Mathematics

Malaviya National Institute of Technology

Jaipur-302017, India

e-mail: maheshgoswami1989@gmail.com

Ravi P. Agarwal

Department of Mathematics

Texas A\&M University

Kingsville 700 University Blvd. Kingsville

TX 78363-8202

e-mail: agarwal@tamuk.edu 